FACTA UNIVERSITATIS (NIŠ)

Ser. Math. Inform. Vol. 35, No 1 (2020), 253-272

https://doi.org/10.22190/FUMI2001253A

\title{
ON THE BASIC STRUCTURES OF DUAL SPACE
}

\author{
Buşra Aktaş, Olgun Durmaz and Halit Gündoğan
}

\author{
(c) 2020 by University of Nišs, Serbia | Creative Commons License: CC BY-NC-ND
}

\begin{abstract}
Topology studies the properties of spaces that are invariant under any continuous deformation. Topology is needed to examine the properties of the space. Fundamentally, the most basic structure required to do math in the space is topology. There exists little information on the expression of the basis and topology on dual space. The main point of the research is to explain how to define the basis and topology on dual space $D^{n}$. Then, we will study the geometric constructions corresponding to the open balls in $D$ and $D^{2}$, respectively.
\end{abstract}

Keywords: dual space; dual numbers; topological structure.

\section{Introduction}

Topology, as a well-determined mathematical field, emerged at the beginning of the 20th century although some isolated conclusions are traced back to a few centuries ago. The term topology belongs to a special mathematical opinion central to the field of mathematics named topology. Topology tells us how components of a group concern spatially with one another. Fundamentally, in the modern version of geometry, the study of all different kinds of spaces can be regarded as topology. The thing that distinguishes different sorts of geometry from each other is in the kinds of transformations that are allowed before you really consider something changed. Topology investigates the properties of spaces that are invariant under any continuous deformation. This is almost the most basic form of geometry available. It is used in nearly all branches of mathematics in one form or another. Besides, topology is applied in biology, computer science, physics, robotics, geography and landscape ecology, fiber art, games, and puzzles. For more details, we refer the readers to $([1],[3]-[10],[18]-[20])$.

Dual numbers were defined by W. K. Clifford (1845-1879) as a tool for his geometrical studies, and their first applications were given by Kotelnikov [15]. Eduard Study [21] used dual numbers and dual vectors in his research on line geometry and kinematics. He proved that there exists a one-to-one correspondence between the

Received September 23, 2019; accepted December 2, 2019

2010 Mathematics Subject Classification. Primary 54A05, 54A10; Secondary 54A99, 47L50 
points of the dual unit sphere in $D^{3}$ and the directed lines of Euclidean 3-space. In 1994, by using dual numbers, Cheng [5] introduced the $C^{H}$ programming language. These numbers play an important role in field theory as well [12]. The most interesting use of dual numbers in field theory can be shown in a series of articles by Wald et al. [22]. Furthermore, Gromov, in a series of articles, applied the dual numbers in several ways: in contractions and analytical continuations of classical groups [13], and then in quantum group formalism [14]. Dual numbers have their application in various fields such as computer modelling of rigid body, mechanism design, kinematics, modelling human body, dynamics, etc. ([11] and [16]). For example, in kinematics, using dual numbers, it is possible to explain the screw theory [17].

The basis and topology concepts of dual space have not been investigated in detail, although dual numbers and dual space are used in many articles about mathematics, kinematics, and physics. In order to study the mathematical structure of dual space, we need its topological structure. Furthermore, there is no order relation on the dual numbers system. In this case, how does the dual absolute value and norm provide triangular inequality? The answer to this question is given by this study.

The main aim of this article is to give the basis and topology concepts on dual space $D^{n}$. The order relation on dual numbers is defined to achieve this aim. By using this order relation, the concepts of dual inner product, norm, and metric are examined again in detail. This study will provide an insight into the structure of dual space.

\section{Basic Concepts}

Let the set of the pair $\left(x, x^{*}\right)$ be

$$
D=\mathbb{R} \times \mathbb{R}=\left\{\bar{x}=\left(x, x^{*}\right) \mid x, x^{*} \in \mathbb{R}\right\} .
$$

Two inner operations and an equality on $D$ are described as follows:

$(i) \oplus: D \times D \rightarrow D$ for $\bar{x}=\left(x, x^{*}\right)$ and $\bar{y}=\left(y, y^{*}\right)$ defined as

$$
\bar{x} \oplus \bar{y}=\left(x+y, x^{*}+y^{*}\right)
$$

is called the addition in $D$.

(ii) $\odot: D \times D \rightarrow D$ for $\bar{x}=\left(x, x^{*}\right)$ and $\bar{y}=\left(y, y^{*}\right)$ defined as

$$
\bar{x} \odot \bar{y}=\left(x y, x y^{*}+x^{*} y\right)
$$

is called the multiplication in $D$.

(iii) For $\bar{x}=\left(x, x^{*}\right)$ and $\bar{y}=\left(y, y^{*}\right)$, if $x=y, x^{*}=y^{*}, \bar{x}$ and $\bar{y}$ are equal, and it is indicated as $\bar{x}=\bar{y}$.

If the two operators and the equality on $D$ with a set of real numbers $\mathbb{R}$ are defined as above, the set $D$ is called the dual numbers system and the element 
$\bar{x}=\left(x, x^{*}\right)$ is called a dual number. For $\bar{x}=\left(x, x^{*}\right) \in D$, the real number $x$ is called the real part of $\bar{x}$, and the real number $x^{*}$ is called the dual part of $\bar{x}$. The dual numbers $(1,0)=1$ and $(0,1)=\varepsilon$ are called the unit element of multiplication operation in $D$, and the dual unit which satisfies the condition that

$$
\varepsilon \neq 0, \varepsilon^{2}=0, \varepsilon 1=1 \varepsilon=\varepsilon,
$$

respectively. If we use the multiplication property and $\varepsilon=(0,1)$, we have the expression $\bar{x}=x+\varepsilon x^{*}$. The set of all dual numbers is written as follows:

$$
D=\left\{\bar{x}=x+\varepsilon x^{*} \mid x, x^{*} \in \mathbb{R}, \varepsilon^{2}=0\right\} .
$$

The set $D$ forms a commutative ring according to the operations

$$
\left(x+\varepsilon x^{*}\right)+\left(y+\varepsilon y^{*}\right)=(x+y)+\varepsilon\left(x^{*}+y^{*}\right)
$$

and

$$
\left(x+\varepsilon x^{*}\right)\left(y+\varepsilon y^{*}\right)=x y+\varepsilon\left(x y^{*}+x^{*} y\right) .
$$

For the dual numbers $\bar{x}=x+\varepsilon x^{*}$ and $\bar{y}=y+\varepsilon y^{*}$, if $y \neq 0$, then the division $\frac{\bar{x}}{\bar{y}}$ is defined as follows:

$$
\frac{\bar{x}}{\bar{y}}=\frac{x}{y}+\varepsilon \frac{x^{*} y-x y^{*}}{y^{2}} .
$$

The absolute value of the dual number $\bar{x}=x+\varepsilon x^{*}$ can be given as

$$
|\bar{x}|_{D}=|x|+\varepsilon \frac{x x^{*}}{|x|},(x \neq 0) .
$$

Clearly, $|\bar{x}|_{D}=0$ if $\bar{x}=0[24]$.

The set of

$$
D^{3}=\left\{\widetilde{x}=\left(\bar{x}_{1}, \bar{x}_{2}, \bar{x}_{3}\right) \mid \bar{x}_{i} \in D, 1 \leq i \leq 3\right\}
$$

gives all triples of dual numbers. The elements of $D^{3}$ are called dual vectors and a dual vector can be represented by

$$
\widetilde{x}=x+\varepsilon x^{*}=\left(x, x^{*}\right),
$$

where $x$ and $x^{*}$ are the vectors of $\mathbb{R}^{3}$. Let us take $\widetilde{x}=x+\varepsilon x^{*}, \widetilde{y}=y+\varepsilon y^{*} \in D^{3}$, and $\bar{\lambda}=\lambda+\varepsilon \lambda^{*} \in D$. Then, the addition and multiplication operations on $D^{3}$ are as below:

$$
\begin{aligned}
\widetilde{x}+\widetilde{y} & =x+y+\varepsilon\left(x^{*}+y^{*}\right), \\
\bar{\lambda} \widetilde{x} & =\lambda x+\varepsilon\left(\lambda x^{*}+\lambda^{*} x\right) .
\end{aligned}
$$

According to these operations, the set $D^{3}$ is a module over the ring $D$ entitled by a $D$-module or dual space $D^{3}[11]$.

The set of dual vectors on $D^{n}$ is represented by

$$
D^{n}=\left\{\widetilde{x}=\left(\bar{x}_{1}, \bar{x}_{2}, \ldots, \bar{x}_{n}\right) \mid \bar{x}_{i} \in D, i=1, \ldots, n\right\} .
$$


These vectors can be given in the form $\widetilde{x}=x+\varepsilon x^{*}=\left(x, x^{*}\right)$, where $x$ and $x^{*}$ are the vectors of $\mathbb{R}^{n}$.

Let $\widetilde{x}=x+\varepsilon x^{*}$ and $\widetilde{y}=y+\varepsilon y^{*}$ be dual vectors of $D^{n}$, and let $\bar{\lambda}=\lambda+\varepsilon \lambda^{*}$ be a dual number. Then we define the following operations that make $D^{n}$ a module called dual space $D^{n}$. These axioms are as follows:

$$
\begin{aligned}
\widetilde{x}+\widetilde{y} & =x+y+\varepsilon\left(x^{*}+y^{*}\right), \\
\bar{\lambda} \widetilde{x} & =\lambda x+\varepsilon\left(\lambda x^{*}+\lambda^{*} x\right) .
\end{aligned}
$$

Formally, a vector space $V$ over the field $F$ together with a function

$$
\langle,\rangle: V \times V \rightarrow F
$$

is called an inner product space satisfying the following three axioms for $x, y, z \in V$ and $\lambda, \mu \in F$ :

i) Symmetric Property:

$$
\langle x, y\rangle=\langle y, x\rangle
$$

ii) Linearity:

$$
\langle\lambda x+\mu y, z\rangle=\lambda\langle x, z\rangle+\mu\langle y, z\rangle
$$

and

$$
\langle x, \lambda y+\mu z\rangle=\lambda\langle x, y\rangle+\mu\langle x, z\rangle .
$$

iii) Positive Definite Property:

$$
\langle x, x\rangle \geq 0
$$

and

$$
\langle x, x\rangle=0 \Leftrightarrow x=0 .
$$

A vector space $V$ is normed vector space if there is a norm function that transforms $V$ to non-negative real numbers, symbolized as $\|x\|$, for all vectors $x, y \in V$ and all scalars $\lambda \in F$, and satisfies the following conditions:

i) $\|x\| \geq 0$ and $\|x\|=0$ if and only if $x=0$,

ii) $\|\lambda x\|=|\lambda| \cdot\|x\|$,

iii) $\|x+y\| \leq\|x\|+\|y\|$ (Triangle inequality).

In 1906, M. Frechet showed that given any non-empty set $W$, a distance function $d: W \times W \rightarrow \mathbb{R}$ may be described. The pair $(W, d)$ is named a metric space, where $W$ is a non-empty set and $d$ is a real valued function on $W \times W$ called a metric that satisfies the following axioms for $x, y, z \in W$ :

d.1) $d(x, y) \geq 0$,

$d .2) d(x, y)=0$ if and only if $x=y$,

$d .3) d(x, y)=d(y, x)$ (Symmetry property),

d.4) $d(x, z) \leq d(x, y)+d(y, z)$ (Triangle inequality). 
Let $(W, d)$ be a metric space. In parallel to $\mathbb{R}^{n}$, the set

$$
\mathbf{B}_{d}(a, r)=\{x \in W \mid d(a, x)<r\}
$$

with $r>0$ is named an open ball with centre $a$ and radius $r$. Similarly, the set

$$
\mathbf{S}_{d}(a, r)=\{x \in W \mid d(a, x) \leq r\}
$$

with $r>0$ is entitled as a closed ball with centre $a$ and radius $r$. In the metric space $(W, d)$, a subset $A \subseteq W$ is called an open set if and only if for all points $a \in A$, there is an $r>0$, such that the open ball $\mathbf{B}_{d}(a, r)$ is a subset of $A$.

Lemma 2.1. An open ball $\mathbf{B}_{d}(a, r)$ in a metric space $(W, d)$ is open.

Definition 2.1. If $W$ is a set, a collection $\beta$ of subsets of $W$ is a basis for a topology on $W$, such that

(1) $\bigcup_{\mathbf{B} \in \beta} \mathbf{B}=W$

(2) $\mathbf{B}_{1} \cap \mathbf{B}_{2}=\bigcup_{\mathbf{B} \in \beta} \mathbf{B}$ for $\forall \mathbf{B}_{1}, \mathbf{B}_{2} \in \beta$, where $\mathbf{B}_{1} \cap \mathbf{B}_{2} \neq \varnothing$.

Let $\beta=\left\{\mathbf{B}_{d}(a, r) \mid a \in W, r \in \mathbb{R}^{+}\right\}$. A collection $\beta$ on $W$ is a topological basis. Assume that the topology obtained from this basis $\beta$ is symbolized as $\tau$. This topology $\tau$ is defined as the metric topology reduced from the metric $d$ on the set $W([2],[18]$ and [23]).

Assume that $A$ and $B$ are any two sets. An ordering for the Cartesian product $A \times B$ is determined as follows:

If not only $\left(a_{1}, b_{1}\right)$ but also $\left(a_{2}, b_{2}\right)$ are the elements of $A \times B$, we can write $\left(a_{1}, b_{1}\right)<$ $\left(a_{2}, b_{2}\right)$ if and only if either

or

1) $a_{1}<_{A} a_{2}$

2) if $a_{1}=a_{2}, b_{1}<_{B} b_{2}$,

where $<_{A}$ and $<_{B}$ are order relations on any two sets $A$ and $B$, respectively. Specifically, let two partially ordered sets $A$ and $B$ be given. The lexicographical order on the Cartesian product $A \times B$ is described as follows:

$$
\left.\left(a_{1}, b_{1}\right) \leq\left(a_{2}, b_{2}\right) \text { if and only if } a_{1}<_{A} a_{2} \text { or (if } a_{1}=a_{2}, b_{1} \leq_{B} b_{2}\right) \text {. }
$$

If $A=B=\mathbb{R}$ is taken into consideration,

$$
\beta=\left\{\left(a_{1} \times b_{1}, a_{2} \times b_{2}\right) \mid a_{1}<a_{2} \text { or }\left(\text { if } a_{1}=a_{2}, b_{1}<b_{2}\right)\right\}
$$

is a basis of $\mathbb{R} \times \mathbb{R}$ with reference to order relation. 


\section{Inner Product, Norm and Metric on Dual Space}

Theorem 3.1. Let $\bar{x}=x+\varepsilon x^{*}$ and $\bar{y}=y+\varepsilon y^{*}$ be dual numbers. In this case, the relation $<_{D}$ defined as

$$
\bar{x}<_{D} \bar{y} \Leftrightarrow x<y \text { or if } x=y, x^{*}<y^{*}
$$

is an order relation on $D$, where $<$ is the order relation on $\mathbb{R}$.

Proof. Let us take $\bar{x}=x+\varepsilon x^{*}, \bar{y}=y+\varepsilon y^{*}, \bar{z}=z+\varepsilon z^{*} \in D$ and

$$
\bar{x}<_{D} \bar{y} \Leftrightarrow x<y \text { or if } x=y, x^{*}<y^{*} .
$$

In this case, by taking account of dual inequality (3.1), it is possible to write the below expressions:

i) if $\bar{x} \neq \bar{y}$, then either $\bar{x}<_{D} \bar{y}$ or $\bar{y}<_{D} \bar{x}$,

ii) if $\bar{x}<_{D} \bar{y}$, then $\bar{x} \neq \bar{y}$,

iii) if $\bar{x}<_{D} \bar{y}$ and $\bar{y}<_{D} \bar{z}$, then $\bar{x}<_{D} \bar{z}$.

Thus, the proof is completed.

Furthermore, for $\bar{x}=x+\varepsilon x^{*}, \bar{y}=y+\varepsilon y^{*}$ and $\bar{z}=z+\varepsilon z^{*} \in D$, the relation $\leq_{D}$ defined as

$$
\bar{x} \leq_{D} \bar{y} \Leftrightarrow x<y \text { or if } x=y, x^{*} \leq y^{*}
$$

provides the following expressions:

i) $\bar{x} \leq_{D} \bar{x}$

ii) if $\bar{x} \leq_{D} \bar{y}$ and $\bar{y} \leq_{D} \bar{x}$, then $\bar{x}=\bar{y}$,

iii) if $\bar{x} \leq_{D} \bar{y}$ and $\bar{y} \leq_{D} \bar{z}$, then $\bar{x} \leq_{D} \bar{z}$,

where $\leq$ is the partial order relation on $\mathbb{R}$. This relation is called the partial order relation on $D$.

In this section, using the above defined order relations on $D$, we will reconsider the concepts of dual norm and metric on $D^{n}$ obtained from the dual inner product.

A dual inner product on dual space $D^{n}$ is a function

$$
\begin{aligned}
\langle,\rangle_{D} & : \quad D^{n} \times D^{n} \rightarrow D, \\
\langle\widetilde{x}, \widetilde{y}\rangle_{D} & =\langle x, y\rangle+\varepsilon\left(\left\langle x, y^{*}\right\rangle+\left\langle x^{*}, y\right\rangle\right),
\end{aligned}
$$

where $\widetilde{x}=x+\varepsilon x^{*}, \widetilde{y}=y+\varepsilon y^{*} \in D^{n}$, and the notation $\langle$,$\rangle is an inner product$ on $\mathbb{R}^{n}$, such that for the dual vectors $\widetilde{x}, \widetilde{y}, \widetilde{z} \in D^{n}$ and the dual numbers $\bar{\lambda}=$ $\lambda+\varepsilon \lambda^{*}, \bar{\mu}=\mu+\varepsilon \mu^{*} \in D$, the following conditions exist:

i) $\langle\widetilde{x}, \widetilde{x}\rangle_{D} \geq_{D} 0$ and $\langle\widetilde{x}, \widetilde{x}\rangle_{D}=0$ if $x=0$.

ii) This inner product $\langle,\rangle_{D}$ satisfies symmetry property, i.e.,

$$
\langle\widetilde{x}, \widetilde{y}\rangle_{D}=\langle\widetilde{y}, \widetilde{x}\rangle_{D} .
$$


iii) This inner product $\langle,\rangle_{D}$ provides bilinear property, i.e.,

$$
\langle\bar{\lambda} \widetilde{x}+\bar{\mu} \widetilde{y}, \widetilde{z}\rangle_{D}=\bar{\lambda}\langle\widetilde{x}, \widetilde{z}\rangle_{D}+\bar{\mu}\langle\widetilde{y}, \widetilde{z}\rangle_{D},
$$

and

$$
\langle\widetilde{x}, \bar{\lambda} \widetilde{y}+\bar{\mu} \widetilde{z}\rangle_{D}=\bar{\lambda}\langle\widetilde{x}, \widetilde{y}\rangle_{D}+\bar{\mu}\langle\widetilde{x}, \widetilde{z}\rangle_{D} .
$$

A dual norm on dual space $D^{n}$ is defined as follows:

$$
\begin{aligned}
& \|\cdot\|_{D}: D^{n} \rightarrow D, \\
& \|\widetilde{x}\|_{D}=\left\{\begin{array}{cc}
0 & , x=0 \\
\|x\|+\varepsilon \frac{\left\langle x, x^{*}\right\rangle}{\|x\|} & , x \neq 0,
\end{array}\right.
\end{aligned}
$$

where $\widetilde{x}=x+\varepsilon x^{*} \in D^{n}$ and $x, x^{*} \in \mathbb{R}^{n}$. The dual norm has the following three properties:

i) For $\widetilde{x} \in D^{n}$,

$$
\|\widetilde{x}\|_{D} \geq_{D} 0
$$

ii) For $\widetilde{x} \in D^{n}$ and $\bar{\lambda} \in D$,

$$
\|\bar{\lambda} \widetilde{x}\|_{D}=|\bar{\lambda}|_{D} \cdot\|\widetilde{x}\|_{D}
$$

iii) We can write the following dual inequalities:

If these conditions $x=0, y \neq 0($ or $x \neq 0, y=0)$ and $\left\langle x^{*}, y\right\rangle \geq 0\left(\right.$ or $\left.\left\langle x, y^{*}\right\rangle \geq 0\right)$ are satisfied, there exists the below dual inequality

$$
\|\widetilde{x}+\widetilde{y}\|_{D} \geq_{D}\|\widetilde{x}\|_{D}+\|\widetilde{y}\|_{D}
$$

In all other cases, it is possible to write the following expression

$$
\|\widetilde{x}+\widetilde{y}\|_{D} \leq_{D}\|\widetilde{x}\|_{D}+\|\widetilde{y}\|_{D}
$$

Here, the third property is called the dual triangle inequality, and these properties are proved by using the definition of dual norm.

A dual distance on dual space $D^{n}$ is a function

$$
\begin{aligned}
\bar{d}: D^{n} \times D^{n} & \rightarrow D, \\
\bar{d}(\widetilde{x}, \widetilde{y}) & =\|\widetilde{x}-\widetilde{y}\|_{D}=\left\{\begin{array}{cc}
0 & , x=y \\
\|x-y\|+\varepsilon \frac{\left\langle x-y, x^{*}-y^{*}\right\rangle}{\|x-y\|} & , x \neq y
\end{array}\right.
\end{aligned}
$$

that satisfies the following conditions for $\widetilde{x}, \widetilde{y}, \widetilde{z} \in D^{n}$ :

i) $\bar{d}(\widetilde{x}, \widetilde{y}) \geq_{D} 0$, and $\bar{d}(\widetilde{x}, \widetilde{y})=0$ if $x=y$.

ii) If the function $\bar{d}$ is taken into consideration, the below symmetry property is satisfied:

$$
\bar{d}(\widetilde{x}, \widetilde{y})=\bar{d}(\widetilde{y}, \widetilde{x}) .
$$


iii) It is possible to say that the dual inequalities exist:

If these conditions $x-y=0, y-z \neq 0($ or $x-y \neq 0, y-z=0)$ and $\left\langle x^{*}-y^{*}, y-z\right\rangle \geq$ 0 (or $\left\langle x-y, y^{*}-z^{*}\right\rangle \geq 0$ ) are satisfied, it is clear that

$$
\bar{d}(\widetilde{x}, \widetilde{z}) \geq_{D} \bar{d}(\widetilde{x}, \widetilde{y})+\bar{d}(\widetilde{y}, \widetilde{z}) .
$$

In all other cases, the following dual inequality is written

$$
\bar{d}(\widetilde{x}, \widetilde{z}) \leq_{D} \bar{d}(\widetilde{x}, \widetilde{y})+\bar{d}(\widetilde{y}, \widetilde{z}) .
$$

\section{Basis and Topology on Dual Space}

The aim in this section is to present how to introduce the concepts of basis and topology on dual space $D^{n}$. Then, the geometric modellings for the open balls of $D$ and $D^{2}$ are shown in detail.

Let $\left(D^{n}, \bar{d}\right)$ be a dual metric space. Given a dual point $\widetilde{a}=a+\varepsilon a^{*} \in D^{n}$ and a dual constant $\bar{r}=r+\varepsilon r^{*}$, where $r>0$, the sets

$$
\begin{aligned}
\widetilde{B}_{\bar{d}}(\widetilde{a}, \bar{r}) & =\left\{\widetilde{x}=x+\varepsilon x^{*} \in D^{n} \mid \bar{d}(\widetilde{x}, \widetilde{a})<{ }_{D} \bar{r}, \bar{r}=r+\varepsilon r^{*}\right\} \\
& =\left\{\begin{array}{c}
\widetilde{x}=x+\varepsilon x^{*} \in D^{n} \mid\|x-a\|<r \text { or if }\|x-a\|=r \\
, \frac{\left\langle x-a, x^{*}-a^{*}\right\rangle}{\|x-a\|}<r^{*}
\end{array}\right\} \cup\left\{a+\varepsilon x^{*}\right\}
\end{aligned}
$$

and

$$
\begin{aligned}
\widetilde{S}_{\bar{d}}(\widetilde{a}, \bar{r}) & =\left\{\widetilde{x}=x+\varepsilon x^{*} \in D^{n} \mid \bar{d}(\widetilde{x}, \widetilde{a}) \leq_{D} \bar{r}, \bar{r}=r+\varepsilon r^{*}\right\} \\
& =\left\{\begin{array}{c}
\widetilde{x}=x+\varepsilon x^{*} \in D^{n} \mid\|x-a\|<r \text { or if }\|x-a\|=r \\
, \frac{\left\langle x-a, x^{*}-a^{*}\right\rangle}{\|x-a\|} \leq r^{*}
\end{array}\right\} \cup\left\{a+\varepsilon x^{*}\right\}
\end{aligned}
$$

are called a dual open ball and dual closed ball with radius $\bar{r}$ and center $\widetilde{a}$, respectively.

Each dual distance function $\bar{d}$ on dual space $D^{n}$ generates a topology $\widetilde{\tau}_{\bar{d}}$ on $D^{n}$, which has a basis on the family of dual open balls

$$
\widetilde{\beta}=\left\{\widetilde{B}_{\bar{d}}(\widetilde{a}, \bar{r}) \mid \widetilde{a} \in D^{n} \text { and } \bar{r}=r+\varepsilon r^{*}\right\} .
$$

Now, we will demonstrate that $\widetilde{\beta}$ is a basis on $D^{n}$ :

i) Due to $\widetilde{a} \in \widetilde{B}_{\bar{d}}(\widetilde{a}, \bar{r})$, it is possible to write $\{\widetilde{a}\} \subset \widetilde{B}_{\bar{d}}(\widetilde{a}, \bar{r})$, and thus we obtain the following equality:

$$
D^{n}=\underset{\widetilde{a} \in D^{n}}{\cup}\{\widetilde{a}\} \subset \underset{\widetilde{B} \in \widetilde{\beta}}{\cup} \widetilde{B}_{\bar{d}}(\widetilde{a}, \bar{r}) \subset D^{n},
$$

i.e.,

$$
\cup_{\widetilde{B} \in \widetilde{\beta}} \widetilde{B}_{\bar{d}}(\widetilde{a}, \bar{r})=D^{n}
$$


ii) For all open balls $\widetilde{B}_{1_{\bar{d}}}=\widetilde{B}_{1_{\bar{d}}}\left(\widetilde{a}_{1}, \bar{r}_{1}\right)$ and $\widetilde{B}_{2_{\bar{d}}}=\widetilde{B}_{2_{\bar{d}}}\left(\widetilde{a}_{2}, \bar{r}_{2}\right)$ except for $\widetilde{B}_{1_{\bar{d}}} \cap \widetilde{B}_{2_{\bar{d}}}=\phi$, we must show the existence of the following equality:

$$
\widetilde{B}_{1_{\bar{d}}}\left(\widetilde{a}_{1}, \bar{r}_{1}\right) \cap \widetilde{B}_{2_{\bar{d}}}\left(\widetilde{a}_{2}, \bar{r}_{2}\right)=\bigcup_{\widetilde{B} \in \widetilde{\beta}} \widetilde{B}_{\bar{d}}(\widetilde{a}, \bar{r}) .
$$

Assume that $\widetilde{y} \in \widetilde{B}_{1_{\bar{d}}} \cap \widetilde{B}_{2_{\bar{d}}}$. Since $\widetilde{y} \in D^{n}$ is the element of not only $\widetilde{B}_{1_{\bar{d}}}$ but also $\widetilde{B}_{2_{\bar{d}}}$, we can write $\bar{d}\left(\widetilde{y}, \widetilde{a}_{1}\right)<_{D} \bar{r}_{1}$ and $\bar{d}\left(\widetilde{y}, \widetilde{a}_{2}\right)<_{D} \bar{r}_{2}$ with respect to the definition of dual open ball, respectively. Thus, dual inequalities are obtained as follows:

$$
\bar{d}\left(\widetilde{y}, \tilde{a}_{1}\right)=\left\{\begin{array}{cl}
0 & , y=a_{1} \\
\left\|y-a_{1}\right\|+\varepsilon \frac{\left\langle y-a_{1}, y^{*}-a_{1}^{*}\right\rangle}{\left\|y-a_{1}\right\|} & , y \neq a_{1}
\end{array}<_{D} \bar{r}_{1}=r_{1}+\varepsilon r_{1}^{*}\right.
$$

and

$$
\bar{d}\left(\widetilde{y}, \widetilde{a}_{2}\right)=\left\{\begin{array}{cl}
0 & , y=a_{2} \\
\left\|y-a_{2}\right\|+\varepsilon \frac{\left\langle y-a_{2}, y^{*}-a_{2}^{*}\right\rangle}{\left\|y-a_{2}\right\|} & , y \neq a_{2}
\end{array}<_{D} \bar{r}_{2}=r_{2}+\varepsilon r_{2}^{*} .\right.
$$

Situation 1. Consider that $y=a_{1}=a_{2}$. This gives the below dual inequalities

$$
\bar{d}\left(\widetilde{y}, \widetilde{a}_{1}\right)=0<_{D} r_{1}+\varepsilon r_{1}^{*}
$$

and

$$
\bar{d}\left(\widetilde{y}, \widetilde{a}_{2}\right)=0<_{D} r_{2}+\varepsilon r_{2}^{*} .
$$

Taking $a_{1}=a_{2}=a$ and dualmin $\left\{\bar{r}_{1}, \bar{r}_{2}\right\}=\bar{r}$ in the above dual inequalities, we find $\bar{d}(\widetilde{y}, \widetilde{a})<_{D} \bar{r}$, that is, $\widetilde{y} \in \widetilde{B}_{\bar{d}}(\widetilde{a}, \bar{r})$.

Situation 2. Let $y=a_{1}$ and $y \neq a_{2}$. We can write

$$
\bar{d}\left(\widetilde{y}, \widetilde{a}_{1}\right)=0<_{D} \bar{r}_{1}
$$

and

$$
\bar{d}\left(\widetilde{y}, \widetilde{a}_{2}\right)<{ }_{D} \bar{r}_{2} \Leftrightarrow\left\|y-a_{2}\right\|<r_{2} \text { or if }\left\|y-a_{2}\right\|=r_{2}, \frac{\left\langle y-a_{2}, y^{*}-a_{2}^{*}\right\rangle}{\left\|y-a_{2}\right\|}<r_{2}^{*} .
$$

When $y=a_{1}=a$ is taken into consideration, we get $\bar{d}(\widetilde{y}, \widetilde{a})<_{D} \bar{r}_{1}$, that is, $\widetilde{y} \in \widetilde{B}_{\bar{d}}\left(\widetilde{a}, \bar{r}_{1}\right)$. Now, let us think $y \neq a_{2}=a$. In this case, we have

$$
\begin{aligned}
\bar{d}(\widetilde{y}, \widetilde{a}) & =\|y-a\|+\varepsilon \frac{\left\langle y-a, y^{*}-a^{*}\right\rangle}{\|y-a\|} \\
& =\left\|y-a_{2}\right\|+\varepsilon \frac{\left\langle y-a_{2}, y^{*}-a^{*}\right\rangle}{\left\|y-a_{2}\right\|} .
\end{aligned}
$$

By taking account of the inequality $\left\|y-a_{2}\right\|<r_{2}$, it is seen that $\bar{d}(\widetilde{y}, \widetilde{a})<_{D} \bar{r}_{2}$, that is, $\widetilde{y} \in \widetilde{B}_{\bar{d}}\left(\widetilde{a}, \bar{r}_{2}\right)$. Assume that $\left\|y-a_{2}\right\|=r_{2}$. Thus, the following inequality is obtained

$$
\frac{\left\langle y-a_{2}, y^{*}-a^{*}\right\rangle}{\left\|y-a_{2}\right\|}<r_{2}^{*}+\frac{\left\langle y-a_{2}, a_{2}^{*}-a^{*}\right\rangle}{\left\|y-a_{2}\right\|}=r_{3}^{*} .
$$


If we use the inequality (4.1), the below expression can be obtained

$$
\bar{d}(\widetilde{y}, \widetilde{a})<_{D} r_{2}+\varepsilon r_{3}^{*}=\bar{r}_{3} .
$$

Therefore, the dual inequality (4.2) implies $\widetilde{y} \in \widetilde{B}_{\bar{d}}\left(\widetilde{a}, \bar{r}_{3}\right)$.

Situation 3. The proof for this situation is as in the situation 2 .

Situation 4. Suppose that $y \neq a_{1}$ and $y \neq a_{2}$. Using the order relation on $D$, it is allowed to write

$$
\left\|\widetilde{y}-\widetilde{a}_{1}\right\|_{D}<_{D} \quad \bar{r}_{1} \Leftrightarrow\left\|y-a_{1}\right\|<r_{1} \text { or if }\left\|y-a_{1}\right\|=r_{1}, \frac{\left\langle y-a_{1}, y^{*}-a_{1}^{*}\right\rangle}{\left\|y-a_{1}\right\|}<r_{1}^{*}
$$

and

$$
\left\|\widetilde{y}-\widetilde{a}_{2}\right\|_{D}<_{D} \quad \bar{r}_{2} \Leftrightarrow\left\|y-a_{2}\right\|<r_{2} \text { or if }\left\|y-a_{2}\right\|=r_{2}, \frac{\left\langle y-a_{2}, y^{*}-a_{2}^{*}\right\rangle}{\left\|y-a_{2}\right\|}<r_{2}^{*} .
$$

In order to analyze this situation, four cases exist. These cases are as follows:

Situation 4.1. By considering $\left\|y-a_{1}\right\|<r_{1}$ and $\left\|y-a_{2}\right\|<r_{2}$, the following inequalities can be written

$$
\|y-a\| \leq\left\|y-a_{1}\right\|+\left\|a_{1}-a\right\|<r_{1}+\left\|a_{1}-a\right\|=r_{11}
$$

and

$$
\|y-a\| \leq\left\|y-a_{2}\right\|+\left\|a_{2}-a\right\|<r_{2}+\left\|a_{1}-a\right\|=r_{22} .
$$

From the inequalities (4.3) and (4.4), we deduce $\|y-a\|<r$, where $\min \left\{r_{11}, r_{22}\right\}=r$. Thus, we can express the dual inequality

$$
\|\widetilde{y}-\widetilde{a}\|_{D}=\|y-a\|+\varepsilon \frac{\left\langle y-a, y^{*}-a^{*}\right\rangle}{\|y-a\|}<_{D} r+\varepsilon r^{*}=\bar{r},
$$

which implies that $\widetilde{y} \in \widetilde{B}_{\bar{d}}(\widetilde{a}, \bar{r})$.

Situation 4.2. Let $\left\|y-a_{1}\right\|<r_{1}$ and if $\left\|y-a_{2}\right\|=r_{2}, \frac{\left\langle y-a_{2}, y^{*}-a_{2}^{*}\right\rangle}{\left\|y-a_{2}\right\|}<r_{2}^{*}$. In this case, we have

$$
\|y-a\| \leq\left\|y-a_{1}\right\|+\left\|a_{1}-a\right\|<r_{1}+\left\|a_{1}-a\right\|=r_{11}
$$

and

$$
\|y-a\| \leq\left\|y-a_{2}\right\|+\left\|a_{2}-a\right\|=r_{2}+\left\|a_{2}-a\right\|=r_{22} .
$$

If we consider the above inequalities, it is easy to see that $\|y-a\|<r$, where $\min \left\{r_{11}, r_{22}\right\}=r$. This immediately implies $\bar{d}(\widetilde{y}, \widetilde{a})<_{D} \bar{r}$, that is, $\widetilde{y} \in \widetilde{B}_{\bar{d}}(\widetilde{a}, \bar{r})$.

Situation 4.3. The proof for this case is as in the situation 4.2.

Situation 4.4. Consider that

$$
\text { if }\left\|y-a_{1}\right\|=r_{1}, \frac{\left\langle y-a_{1}, y^{*}-a_{1}^{*}\right\rangle}{\left\|y-a_{1}\right\|}<r_{1}^{*}
$$


and

$$
\text { if }\left\|y-a_{2}\right\|=r_{2}, \frac{\left\langle y-a_{2}, y^{*}-a_{2}^{*}\right\rangle}{\left\|y-a_{2}\right\|}<r_{2}^{*} .
$$

Similarly, we can write the following inequalities:

$$
\|y-a\| \leq\left\|y-a_{1}\right\|+\left\|a_{1}-a\right\|=r_{1}+\left\|a_{1}-a\right\|=r_{11}
$$

and

$$
\|y-a\| \leq\left\|y-a_{2}\right\|+\left\|a_{2}-a\right\|=r_{2}+\left\|a_{2}-a\right\|=r_{22} .
$$

It is seen from the above inequalities that $\|y-a\| \leq r$, where $\min \left\{r_{11}, r_{22}\right\}=r$. Therefore, we have the expressions below:

$$
\|y-a\|<r \text { or }\|y-a\|=r .
$$

By considering $\|y-a\|<r$, it is certain that $\widetilde{y} \in \widetilde{B}_{\bar{d}}(\widetilde{a}, \bar{r})$. Now, let us assume that $\|y-a\|=r$. The following inequalities can be written

$$
\frac{\left\langle y-a, y^{*}-a^{*}\right\rangle}{\|y-a\|}<\frac{r_{1} r_{1}^{*}+\left\langle y-a_{1}, a_{1}^{*}-a^{*}\right\rangle+\left\langle a_{1}-a, y^{*}-a^{*}\right\rangle}{\|y-a\|}=r_{11}^{*}
$$

and

$$
\frac{\left\langle y-a, y^{*}-a^{*}\right\rangle}{\|y-a\|}<\frac{r_{2} r_{2}^{*}+\left\langle y-a_{2}, a_{2}^{*}-a_{0}^{*}\right\rangle+\left\langle a_{2}-a, y^{*}-a^{*}\right\rangle}{\|y-a\|}=r_{22}^{*} .
$$

If we choose $r^{*}=\min \left\{r_{11}^{*}, r_{22}^{*}\right\}$, the below inequality can be written:

$$
\frac{\left\langle y-a, y^{*}-a^{*}\right\rangle}{\|y-a\|}<r^{*} .
$$

From the inequality (4.5) and $\|y-a\|=r$, we obtain $\widetilde{y} \in \widetilde{B}_{\bar{d}}(\widetilde{a}, \bar{r})$.

Consequently, considering four situations together, it is clear that

$$
\widetilde{y} \in \underset{\widetilde{B} \in \widetilde{\beta}}{\cup} \widetilde{B}_{\bar{d}}(\widetilde{a}, \bar{r}) .
$$

On the contrary, assume that $\widetilde{y} \in \underset{\widetilde{B} \in \widetilde{\beta}}{\cup} \widetilde{B}_{\bar{d}}(\widetilde{a}, \bar{r})$. Thus, there exist $\bar{r}_{0}=r_{0}+\varepsilon r_{0}^{*} \in D$ and $\widetilde{a}_{0}=a_{0}+\varepsilon a_{0}^{*} \in D^{n}$, such that it is possible to write $\widetilde{y} \in \widetilde{B}_{\bar{d}}\left(\widetilde{a}_{0}, \bar{r}_{0}\right)$, where $r_{0}>0$. In this case, we have the expression

$$
\bar{d}\left(\widetilde{y}, \widetilde{a}_{0}\right)=\left\{\begin{array}{cl}
0 & , y=a_{0} \\
\left\|y-a_{0}\right\|+\varepsilon \frac{\left\langle y-a_{0}, y^{*}-a_{0}^{*}\right\rangle}{\left\|y-a_{0}\right\|} & , y \neq a_{0}
\end{array}<_{D} r_{0}+\varepsilon r_{0}^{*} .\right.
$$

Therefore, we need to study the following cases:

Case A: Let us consider that $y \neq a_{0}$. By using the order relation on $D$, we can write

$$
\left\|y-a_{0}\right\|<r_{0} \text { or if }\left\|y-a_{0}\right\|=r_{0}, \frac{\left\langle y-a_{0}, y^{*}-a_{0}^{*}\right\rangle}{\left\|y-a_{0}\right\|}<r_{0}^{*} .
$$


Case A.1. Assume that $\left\|y-a_{0}\right\|<r_{0}$. We have the following inequalities:

$$
\left\|y-a_{1}\right\| \leq\left\|y-a_{0}\right\|+\left\|a_{0}-a_{1}\right\|<r_{0}+\left\|a_{0}-a_{1}\right\|=r_{1}
$$

and

$$
\left\|y-a_{2}\right\| \leq\left\|y-a_{0}\right\|+\left\|a_{0}-a_{2}\right\|<r_{0}+\left\|a_{0}-a_{2}\right\|=r_{2} .
$$

If $y=a_{1}$ and $y=a_{2}$ are taken, then it is obvious that

$$
\widetilde{y} \in \widetilde{B}_{1 \bar{d}} \cap \widetilde{B}_{2 \bar{d}} .
$$

If $y \neq a_{1}$ and $y \neq a_{2}$ are considered, due to

$$
\left\|y-a_{1}\right\|<r_{1}
$$

and

$$
\left\|y-a_{2}\right\|<r_{2}
$$

it is clear that

$$
\widetilde{y} \in \widetilde{B}_{1 \bar{d}} \cap \widetilde{B}_{2 \bar{d}}
$$

We can express $\widetilde{y} \in \widetilde{B}_{1 \bar{d}} \cap \widetilde{B}_{2 \bar{d}}$ by means of similar calculations for the situations $y=a_{2}, y \neq a_{1}$ and $y=a_{1}, y \neq a_{2}$.

Case A.2. Suppose that if $\left\|y-a_{0}\right\|=r_{0}, \frac{\left\langle y-a_{0}, y^{*}-a_{0}^{*}\right\rangle}{\left\|y-a_{0}\right\|}<r_{0}^{*}$. Due to

$$
\left\|y-a_{1}\right\| \leq\left\|y-a_{0}\right\|+\left\|a_{0}-a_{1}\right\|=r_{0}+\left\|a_{0}-a_{1}\right\|=r_{1}
$$

and

$$
\left\|y-a_{2}\right\| \leq\left\|y-a_{0}\right\|+\left\|a_{0}-a_{2}\right\|=r_{0}+\left\|a_{0}-a_{2}\right\|=r_{2},
$$

the following inequalities are obtained

$$
\left\|y-a_{1}\right\| \leq r_{1}
$$

and

$$
\left\|y-a_{2}\right\| \leq r_{2} .
$$

By taking account of the above inequalities, we have four cases:

Case A.2.1. Let us think that $\left\|y-a_{1}\right\|<r_{1}$ and $\left\|y-a_{2}\right\|<r_{2}$. Then we obtain

$$
\widetilde{y} \in \widetilde{B}_{1 \bar{d}} \cap \widetilde{B}_{2 \bar{d}} .
$$

Case A.2.2. Let us consider that $\left\|y-a_{1}\right\|=r_{1}$ and $\left\|y-a_{2}\right\|<r_{2}$. From the second expression, we attain

$$
\widetilde{y} \in \widetilde{B}_{2 \bar{d}}
$$

On the other hand, the inequality

$$
\frac{\left\langle y-a_{1}, y^{*}-a_{1}^{*}\right\rangle}{\left\|y-a_{1}\right\|}<\frac{r_{0} r_{0}^{*}+\left\langle a_{0}-a_{1}, y^{*}-a_{1}^{*}\right\rangle+\left\langle y-a_{0}, a_{0}^{*}-a_{1}^{*}\right\rangle}{\left\|y-a_{1}\right\|}=r_{1}^{*}
$$


can be written. Because of $\left\|y-a_{1}\right\|=r_{1}$ and the inequality (4.8), it is possible to write

$$
\widetilde{y} \in \widetilde{B}_{1 \bar{d}}
$$

Thus, it is easy to see that

$$
\widetilde{y} \in \widetilde{B}_{1 \bar{d}} \cap \widetilde{B}_{2 \bar{d}}
$$

Case A.2.3. The proof for this case is as in the case A.2.2.

Case A.2.4. Assume that $\left\|y-a_{1}\right\|=r_{1}$ and $\left\|y-a_{2}\right\|=r_{2}$. In this case, the following inequalities are calculated

$$
\frac{\left\langle y-a_{1}, y^{*}-a_{1}^{*}\right\rangle}{\left\|y-a_{1}\right\|}<\frac{r_{0} r_{0}^{*}+\left\langle a_{0}-a_{1}, y^{*}-a_{1}^{*}\right\rangle+\left\langle y-a_{0}, a_{0}^{*}-a_{1}^{*}\right\rangle}{\left\|y-a_{1}\right\|}=r_{1}^{*}
$$

and

$$
\frac{\left\langle y-a_{2}, y^{*}-a_{2}^{*}\right\rangle}{\left\|y-a_{2}\right\|}<\frac{r_{0} r_{0}^{*}+\left\langle a_{0}-a_{2}, y^{*}-a_{2}^{*}\right\rangle+\left\langle y-a_{0}, a_{0}^{*}-a_{2}^{*}\right\rangle}{\left\|y-a_{2}\right\|}=r_{2}^{*} .
$$

If we take account of the above inequalities, these imply that

$$
\widetilde{y} \in \widetilde{B}_{1 \bar{d}} \cap \widetilde{B}_{2 \bar{d}} .
$$

Case B: Let us assume that $y=a_{0}$. By revisiting the dual inequality (4.6), we have

$$
\bar{d}\left(\widetilde{y}, \widetilde{a}_{0}\right)=0<_{D} r_{0}+\varepsilon r_{0}^{*} .
$$

Case B.1. If $a_{1}=a_{2}=a_{0}$ is considered, we obtain the following dual inequalities:

$$
\bar{d}\left(\widetilde{y}, \widetilde{a}_{1}\right)=0<{ }_{D} \bar{r}_{1}
$$

and

$$
\bar{d}\left(\widetilde{y}, \widetilde{a}_{2}\right)=0<_{D} \bar{r}_{2}
$$

Thus, we have

$$
\widetilde{y} \in \widetilde{B}_{1 \bar{d}} \cap \widetilde{B}_{2 \bar{d}}
$$

Case B.2. We can make similar computations for the situations $a_{1}=a_{0}, a_{2} \neq$ $a_{0}$ and $a_{2}=a_{0}, a_{1} \neq a_{0}$.

Case B.3. Consider that $a_{0} \neq a_{1}$ and $a_{0} \neq a_{2}$. Because of $y=a_{0}$, the following equalities can be written:

$$
\left\|y-a_{1}\right\|=\left\|a_{0}-a_{1}\right\|
$$

and

$$
\left\|y-a_{2}\right\|=\left\|a_{0}-a_{2}\right\| .
$$

Case B.3.1. For $0<\delta_{1}, \delta_{2}<1$, if we choose

$$
r_{1}=\left\|a_{0}-a_{1}\right\|+\delta_{1}
$$


and

$$
r_{2}=\left\|a_{0}-a_{2}\right\|+\delta_{2}
$$

it is seen that

$$
\left\|y-a_{1}\right\|<r_{1}
$$

and

$$
\left\|y-a_{2}\right\|<r_{2}
$$

Thereby, the below expression is attained

$$
\widetilde{y} \in \widetilde{B}_{1 \bar{d}} \cap \widetilde{B}_{2 \bar{d}} .
$$

Case B.3.2. Let us take $r_{1}=\left\|a_{0}-a_{1}\right\|+\delta_{1}$ and $r_{2}=\left\|a_{0}-a_{2}\right\|$. In this case, we have the following expressions:

$$
\left\|y-a_{1}\right\|<r_{1}
$$

and

$$
\left\|y-a_{2}\right\|=r_{2} \text {. }
$$

The first inequality implies that $\widetilde{y} \in \widetilde{B}_{1 \bar{d}}$. On the other hand, we can write

$$
\frac{\left\langle y-a_{2}, y^{*}-a_{2}^{*}\right\rangle}{\left\|y-a_{2}\right\|}=\left\|y^{*}-a_{2}^{*}\right\| \cdot \cos \varphi
$$

where the angle $\varphi$ is between the vectors $y-a_{2}$ and $y^{*}-a_{2}^{*}$. Since $-1 \leq \cos \varphi \leq 1$, we can find $r_{2}^{*} \in \mathbb{R}$, such that

$$
\frac{\left\langle y-a_{2}, y^{*}-a_{2}^{*}\right\rangle}{\left\|y-a_{2}\right\|}<r_{2}^{*} .
$$

From the inequality (4.10) and $\left\|y-a_{2}\right\|=r_{2}$, we deduce $\widetilde{y} \in \widetilde{B}_{2 \bar{d}}$. Thus, considering the above statements, it is obvious that

$$
\widetilde{y} \in \widetilde{B}_{1 \bar{d}} \cap \widetilde{B}_{2 \bar{d}}
$$

Case B.3.3. The proof for this case is as in the Case B.3.2.

Case B.3.4. Assume that $r_{1}=\left\|a_{0}-a_{1}\right\|$ and $r_{2}=\left\|a_{0}-a_{2}\right\|$. Therefore, there are the following inequalities:

$$
\frac{\left\langle y-a_{1}, y^{*}-a_{1}^{*}\right\rangle}{\left\|y-a_{1}\right\|}<r_{1}^{*}
$$

and

$$
\frac{\left\langle y-a_{2}, y^{*}-a_{2}^{*}\right\rangle}{\left\|y-a_{2}\right\|}<r_{2}^{*},
$$

where $r_{1}^{*}, r_{2}^{*} \in \mathbb{R}$. Hence, $\widetilde{y} \in \widetilde{B}_{1 \bar{d}} \cap \widetilde{B}_{2 \bar{d}}$. 
Consequently, since

$$
\widetilde{B}_{1 \bar{d}}\left(\widetilde{a}_{1}, \bar{r}_{1}\right) \cap \widetilde{B}_{2 \bar{d}}\left(\widetilde{a}_{2}, \bar{r}_{2}\right) \subset \underset{\widetilde{B} \in \widetilde{\beta}}{\cup} \widetilde{B}_{\bar{d}}(\widetilde{a}, \bar{r})
$$

and

$$
\underset{\widetilde{B} \in \widetilde{\beta}}{\cup} \widetilde{B}_{\bar{d}}(\widetilde{a}, \bar{r}) \subset \widetilde{B}_{1 \bar{d}}\left(\widetilde{a}_{1}, \bar{r}_{1}\right) \cap \widetilde{B}_{2 \bar{d}}\left(\widetilde{a}_{2}, \bar{r}_{2}\right),
$$

we can write

$$
\widetilde{B}_{1 \bar{d}}\left(\widetilde{a}_{1}, \bar{r}_{1}\right) \cap \widetilde{B}_{2 \bar{d}}\left(\widetilde{a}_{2}, \bar{r}_{2}\right)=\underset{\widetilde{B} \in \widetilde{\beta}}{\cup} \widetilde{B}_{\bar{d}}(\widetilde{a}, \bar{r})
$$

In this case, from $(i)$ and $(i i)$, it is seen that $\widetilde{\beta}$ is a basis on $D^{n}$. Consider that the topology obtained from this basis $\widetilde{\beta}$ is indicated by $\widetilde{\tau}_{\bar{d}}$. The topology $\widetilde{\tau}_{\bar{d}}$ is called the dual metric topology reduced from the dual distance function $\bar{d}$ on the set $D^{n}$.

Now, the geometric modellings that correspond to the dual open balls on $D$ and $D^{2}$ will be investigated. Firstly, on $D$, we study the geometric modelling for the dual open ball $\widetilde{B}(0, \bar{r})$ with center origin and dual radius $\bar{r}=r+\varepsilon r^{*}$, where $r>0$ :

$$
\begin{aligned}
\widetilde{B}(0, \bar{r}) & =\left\{\widetilde{x} \in D \mid \bar{d}(\bar{x}, 0)<_{D} \bar{r}\right\} \\
& =\left\{\bar{x}=x+\left.\varepsilon x^{*} \in D|| \bar{x}\right|_{D}<_{D} \bar{r}\right\} \\
& =\left\{\widetilde{x}=x+\varepsilon x^{*} \in D|| x \mid<r \text { or if }|x|=r, \frac{x \cdot x^{*}}{|x|}<r^{*}\right\} \cup\left\{0+\varepsilon x^{*}\right\} .
\end{aligned}
$$

The geometric modelling of the dual open ball $\widetilde{B}(0, \bar{r})$ is as follows:

i) Let us consider that $|x|<r$. In this case, we have $-r<x<r$.

ii) Let us take $|x|=r$. In this situation, we get

$$
x=r \Rightarrow x^{*}<r^{*}
$$

and

$$
x=-r \Rightarrow x^{*}>-r^{*} \text {. }
$$

Thus, if we take the situations $(i)$ and $(i i)$ together, the geometric modellings of the dual open balls are indicated by Fig.1. according to the situations of $r^{*}$. 

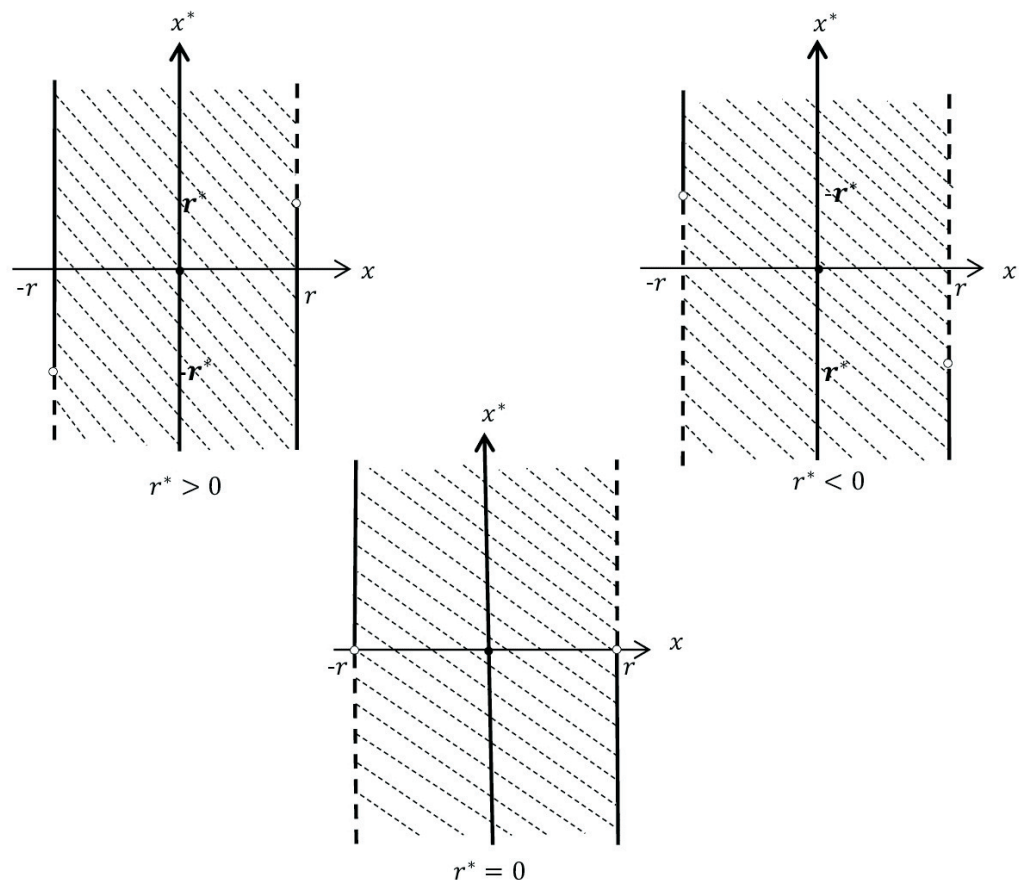

Fig. 1. The geometric modellings in $\mathbb{R} \times \mathbb{R}$, for the dual open balls of $D$.

Then, on $D^{2}$, we demonstrate the geometric structure corresponding to the dual open ball $\widetilde{B}(0, \bar{r})$ with center origin and dual radius $\bar{r}=r+\varepsilon r^{*}$, where $r>0$ :

$$
\begin{aligned}
\widetilde{B}(0, \bar{r}) & =\left\{\widetilde{x} \in D^{2} \mid \bar{d}(\widetilde{x}, 0)<_{D} \bar{r}\right\} \\
& =\left\{\widetilde{x}=x+\varepsilon x^{*} \in D^{2} \mid\|\widetilde{x}\|_{D}<_{D} \bar{r}\right\} \\
& =\left\{\widetilde{x}=x+\varepsilon x^{*} \in D^{2} \mid\|x\|<r \text { or if }\|x\|=r, \frac{\left\langle x, x^{*}\right\rangle}{\|x\|}<r^{*}\right\} \cup\left\{0+\varepsilon x^{*}\right\} .
\end{aligned}
$$

i) Let us take $\|x\|<r$. This inequality states the interior of the circle with radius $r . x^{*}$ takes any value in $\mathbb{R}^{2}$.

ii) Assume that $\|x\|=r$. Thus, $\left\|x^{*}\right\| \cdot \cos \theta<r^{*}$ is obtained, where $\theta$ is the angle between the vectors $x$ and $x^{*}$. To see the modellings of $D^{2}$ in $\mathbb{R}^{3}$, let us take $x^{*}=\left(x_{1}^{*}, 0\right)$. In this case, we can write

$$
\left|x_{1}^{*}\right| \cdot \cos \theta<r^{*} .
$$

Because $-1 \leq \cos \theta \leq 1$, we have the following equality:

$$
\cos \theta=\left\{\begin{array}{cc}
-\lambda^{2} & ,-1 \leq \cos \theta<0 \\
\lambda^{2} & , 0<\cos \theta \leq 1 \\
0 & , \cos \theta=0
\end{array}\right.
$$


where $0<\lambda \leq 1$. According to the situations of $r^{*}$ and $\cos \theta$, we will investigate the geometric modellings of $\widetilde{B}(0, \bar{r})$ :

Case 1. Let us consider $r^{*}>0$. For $0 \neq \mu \in \mathbb{R}, r^{*}=\mu^{2}$ can be written. From the inequality (4.11), it is obvious that

$$
\left|x_{1}^{*}\right| \cdot \cos \theta<\mu^{2} .
$$

If $\cos \theta=\lambda^{2}$ is taken into consideration, the following inequality is written:

$$
\left|x_{1}^{*}\right|<\frac{\mu^{2}}{\lambda^{2}} .
$$

In this situation, the geometric modelling of $\widetilde{B}(0, \bar{r})$ is shown by Fig.2. If $\cos \theta=$ $-\lambda^{2}$ is taken, it is possible to attain the below inequality:

$$
\left|x_{1}^{*}\right|>-\frac{\mu^{2}}{\lambda^{2}} .
$$

For $\forall x_{1}^{*} \in \mathbb{R}$, the above situation is provided. Geometric modelling of this situation is described by Fig.3. Also, if $\cos \theta=0$ is taken into consideration, the following inequality is obtained

$$
\left|x_{1}^{*}\right| \cdot 0<\mu^{2} .
$$

For $\forall x_{1}^{*} \in \mathbb{R}$, the above inequality can be written. The geometric modelling for this situation is as in Fig.3.
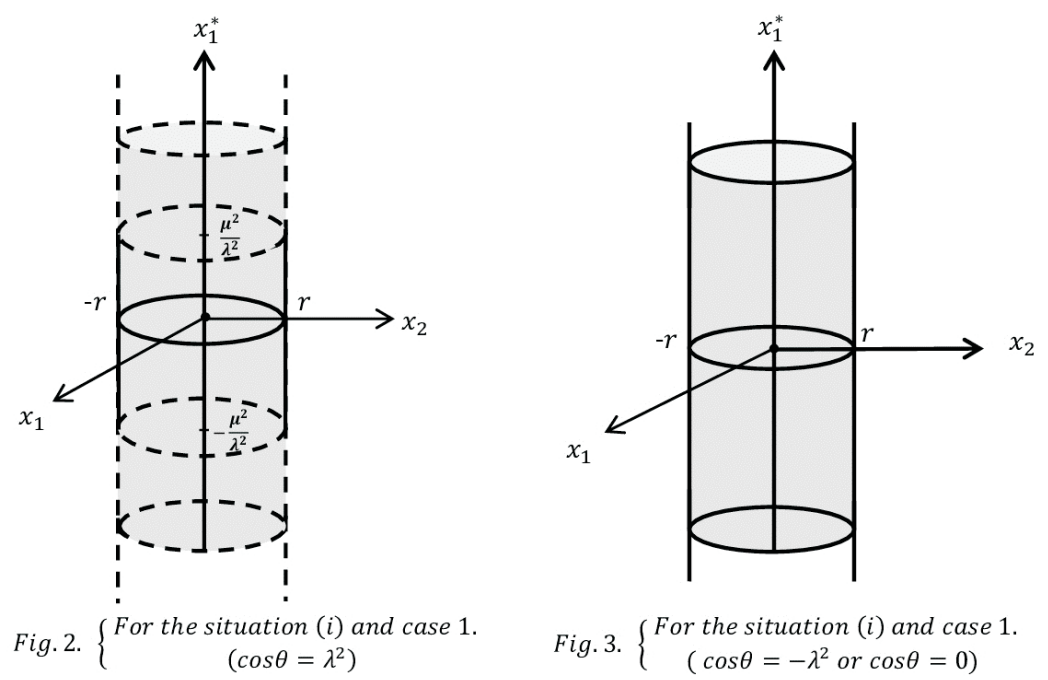

Case 2. Suppose that $r^{*}<0$. We can write $r^{*}=-\mu^{2}$, for $0 \neq \mu \in \mathbb{R}$. If we use the inequality (4.11), we have

$$
\left|x_{1}^{*}\right| \cdot \cos \theta<-\mu^{2} .
$$


This is only valid in the event of $\cos \theta=-\lambda^{2}$. In that case, the following inequality

$$
\left|x_{1}^{*}\right|>\frac{\mu^{2}}{\lambda^{2}}
$$

is obtained. Thus, the geometric modelling of this situation is represented by Fig.4.

Case 3. Finally, let us take $r^{*}=0$. From the inequality (4.11), we have

$$
\left|x_{1}^{*}\right| \cdot \cos \theta<0 .
$$

Similarly, this is only possible in case of $\cos \theta=-\lambda^{2}$. So,

$$
\left|x_{1}^{*}\right|>0
$$

is obtained and the geometric modelling of this situation is showed in Fig.5.

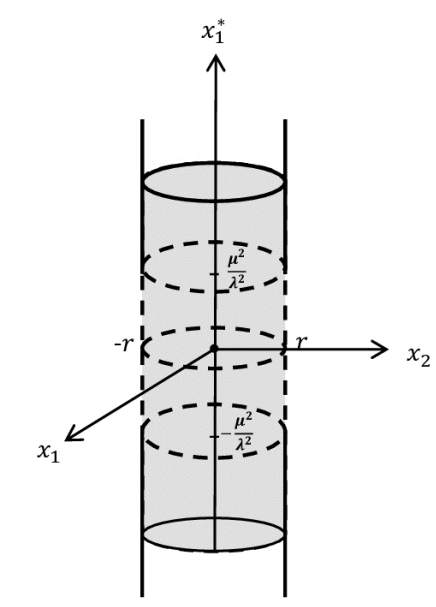

Fig. 4. For the situation (i) and case 2.

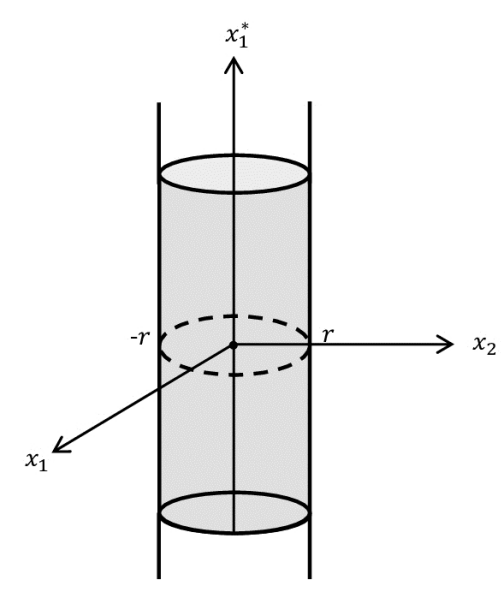

Fig. 5. For the situation (i) and case 3.

Conclusion 4.1. Throughout this paper, the order relations on $D$ is introduced with reference to the lexicographical order relation on the Cartesian product. According to this order relation on $D$, the concepts of dual inner product, norm, and metric have been investigated. After that, by using the order relation, the notation of dual basis has been studied in detail and the geometric modelings for the open balls of $D$ and $D^{2}$ have been given in the last section, respectively.

Acknowledgement 1. The first and the second author would like to thank TUBITAK (The Scientific and Technological Research Council of Turkey) for their financial support during their PhD studies. 


\section{REF E R E N C ES}

1. C. C. Adams, The Knot Book: An Elementary Introduction to the Mathematical Theory of Knots, American Mathematical Society, 2004.

2. N. Bourbaki, Elements of Mathematics, General Topology Chapters 1-4, SpringerVerlag Berlin Heidelberg New York, 1989.

3. A. D. Cambou and N. Menon, Three-Dimensional Structure of a Sheet Crumpled into a Ball, Proc. Natl. Acad. Sci. USA, 108(36) (2011), 14741-14745.

4. G. Carlsson, Topology and Data, Bulletin of the American Mathematical Society, 46(2) (2009), 255-308.

5. H. H. Cheng, Programming with Dual Numbers and its Applications in Mechanisms Design, Eng. Comput, 10(4) (1994), 212-229.

6. W. K. Clifford, Preliminary Sketch of Biquaternions, London Math. Soc., 4(1) (1871), 381-395.

7. J. J. Craig, Introduction to Robotics: Mechanics and Control, 3rd Ed. Prentice-Hall, 2004.

8. M. Dekker, The Shape of Space: How to Visualize Surfaces and Three-Dimensional Manifolds 2nd Edition, C RC Press, 2002.

9. E. Eckman, Connect the Shapes Crochet Motifs: Creative Techniques for Joining Motifs of All Shapes, Storey Publishing, 2012.

10. M. Faber, Invitation to Topological Robotics, European Mathematical Society, University of Durham, Durham, England, 2008.

11. I. S. Fischer, Dual-Number Methods in Kinematics, Statics and Dynamics, CRC Press LLC, 1999.

12. A. M. Frydryszak, Dual Numbers and Supersymmetric Mechanics, Czech. J. Phys., 55(11) (2005), 1409-1414.

13. N. A. Gromov, Contractions and Analytical Continuations of Classical Groups: Unified Approach, Komi Science Center, Syktyvkar (in Russian), 1990.

14. N. A. Gromov, The Matrix Quantum Unitary Cayley-Klein Groups, J. Phys. A: Math. Gen, 26(1) (1993), L5-L8.

15. A. P. Kotelnikov, Screw Calculus and Some of its Applications in Geometry and Mechanics, Annals of the Imperial University, Kazan, 1895.

16. S. Li and Q. J. Ge, Rational Bezier Line-Symmetric Motion, Trans. ASME J. Mech. Design, 127(2) (2005), 222-226.

17. J. M. McCarthy, An Introduction to Theoretical Kinematics, the MIT Press, London, 1990.

18. S. A. Naimpally and J. F. Peters, Topology with Applications, Topological Spaces Near and Far, World Scientific Publishing Co. Pte. Ltd., Hackensack, NJ, 2013.

19. B. M. Stadler, P. F. Stadler, G. P. Wagner and W. Fontana, The Topology of the Possible: Formal Spaces Underlying Patterns of Evolutionary Change, Journal of Theoretical Biology, 213(2) (2001), 241-274.

20. C. Stephenson, D. Lyon and A. Hübler, Topological Properties of a Self-Assembled Electrical Network via Ab Initio Calculation, Sci. Rep., 7:41621 (2017), 1-8. 
21. E. Study, Geometrie der Dynamen, Druck und Verlag von B.G. Teubner, Leipzig, 1903.

22. R. M. Wald, A new Type of Gauge Invariance for a Collection of Massles Spin-2 Fields, II. Geometrical Interpretation. Class. Quant. Grav. 4(5) (1987b), 1279-1316.

23. S. Willard, General Topology, Addison-Wesley, 1970.

24. G. R. Veldkamp, On the Use of Dual Numbers, Vectors and Matrices in Instantaneous Spatial Kinematics, Mechanism and Machine Theory, 11 (1976), 141-156.

Buşra Aktaş

Faculty of Science and Arts

Department of Mathematics

University of Kırıkkale

71450-Yahşihan, Kırıkkale, Turkey

baktas6638@gmail.com

\section{Olgun Durmaz}

Faculty of Science

Department of Mathematics

University of Atat ürk

25240-Yakutiye, Erzurum, Turkey

olgun.durmaz@atauni.edu.tr

Halit Gündoğan

Faculty of Science and Arts

Department of Mathematics

University of Kırıkkale

71450-Yahşihan, Kırıkkale, Turkey

hagundogan@hotmail.com 